\title{
Tsallis scaling and the long-range Ising chain: A transfer matrix approach
}

\author{
R. F. S. Andrade and S. T. R. Pinho \\ Instituto de Física, Universidade Federal da Bahia, 40210-340 Salvador, Brazil \\ (Received 8 June 2004; revised manuscript received 3 December 2004; published 25 February 2005)
}

\begin{abstract}
A numerically efficient transfer matrix (TM) approach is introduced to investigate the long-range Ising spin chain. Results obtained within this procedure are primarily used to verify the Tsallis scaling hypothesis for long-range systems with an $\alpha$ power-law decay of the interaction constants, both in the extensive $(\alpha>1)$ and nonextensive $(\alpha<1)$ regimes. Results for finite-size systems, taking into account all interactions between spins up to 24 sites apart, show that the conjecture is satisfied with a very good precision (less than $0.004 \%$ ) for all temperature intervals. This TM procedure is further used to investigate several other thermodynamic and critical properties of this system, and it may also be extended to similar one-dimensional long-range systems.
\end{abstract}

DOI: 10.1103/PhysRevE.71.026126

PACS number(s): 05.50.+q, 05.70.Fh, 75.10.Pq

\section{INTRODUCTION}

The long-range Ising chain constitutes a classical challenge that has attracted the attention of physicists for many decades. In its more common version, each spin $\sigma_{i}$ interacts with all other spins on the chain mediated by coupling constants $J_{r}=J / r^{\alpha}$, where $r$ is the distance between the interacting spins measured in integer number of lattice spacings. Despite the absence of a closed solution, much is known about this system. Existence theorems for phase transitions have been obtained by a large number of authors, e.g., [1-4]. On the other hand, sophisticated numerical schemes have led to very precise estimates for values of the critical temperature, $T_{c}$, and critical exponents, as reported, e.g., in [5-9]. This large number of contributions have indicated that, for $\alpha>2$, the system shows only a disordered phase, $\forall T$ [4]. Phase transition at finite temperature is found for $1<\alpha \leqslant 2$ $[1,2]$, with the presence of an ordered phase when $T<T_{c}$. For $0 \leqslant \alpha \leqslant 1$, the system is nonextensive [10] and it has a single ordered phase $\forall T$. Classical mean-field exponents are found when $1<\alpha \leqslant 1.5$ [4], while for $\alpha=2$ a discontinuous magnetization at $T_{c}$ is observed [2].

The purpose of this work is to investigate the validity of a conjecture raised by Tsallis [11] to the Ising long-range chain, using a first-principles solution that comes as close as possible to the exact one. Our solution is provided by a transfer matrix (TM) approach that leads to numerical results taking into account the long-range interaction between spins up to a certain distance $g$ apart. It has been optimized regarding both the required space to store the energy values for all distinct spin configurations and the successive increase in the value of $g$ as well as avoiding the numerical evaluation of the TM largest eigenvalue.

In 1995, Tsallis conjectured a universal scaling scheme for thermodynamic functions that should be valid for a large class of both extensive and nonextensive long-range models. For the later models, the energy per degree of freedom diverges, so that the usual intensive energies are devoid of significance. The Tsallis scaling conjecture (TS) [10,11] states that any intensive energylike thermodynamic property, e.g., in the case of magnetic systems, the free Gibbs energy, $f(T, H, N)=F(T, H, N) / N$, of a finite system of $N$ constituents, in a $d$-dimensional space, with long-range interaction decaying with the distance $r$ between particles as $1 / r^{\alpha}$, can be properly described by an $N$-independent function

$$
\tilde{f}(\tilde{T}=T / \tilde{N}, \tilde{H}=H / \tilde{N})=f(T, H, N) / \widetilde{N}
$$

with the help of a scaling variable $\tilde{N}$, defined as

$$
\tilde{N}=\frac{N^{1-\alpha / d}-\alpha / d}{1-\alpha / d} .
$$

When $N \rightarrow \infty$, we obtain limit values for $\tilde{N}$ as a function of $d$ and $\alpha$,

$$
\tilde{N}=\left\{\begin{array}{lll}
\frac{\alpha / d}{\alpha / d-1} & \text { if } & \alpha / d>1 \\
\ln N & \text { if } & \alpha / d=1 \\
\frac{1}{1-\alpha / d} N^{1-\alpha / d} & \text { if } & 0 \leqslant \alpha / d<1 .
\end{array}\right.
$$

According to the same conjecture, other intensive thermodynamic functions depending on the temperature $T$, the magnetic field $H$, and also on $N$, like the entropy $s$, specific heat $c$, and magnetization $m$, admit $N$-independent related functions defined as

$$
\begin{aligned}
\widetilde{s}(\widetilde{T}, \widetilde{H}) & =s(T, H, N), \\
\widetilde{c}(\widetilde{T}, \widetilde{H}) & =c(T, H, N), \\
\widetilde{m}(\widetilde{T}, \widetilde{H}) & =m(T, H, N) .
\end{aligned}
$$

TS takes into account the divergence in the definition of usual intensive quantities, as the free energy $f$, for nonextensive models $(\alpha / d \leqslant 1)$, and generalizes the ad hoc normalization procedure to treat mean-field models, which simply consists of replacing the single-coupling constant for all pairs of particles $J$ by $J / N$. This particular value is recovered in definition (2) when $\alpha \rightarrow 0$. For extensive long-range models $(\alpha / d>1)$, the usual intensive energies as $f$ will reach an $N$-independent value when $N \rightarrow \infty$, but the scaling procedure shows a much faster convergence to $\tilde{f}$, valid for finite-size systems. 


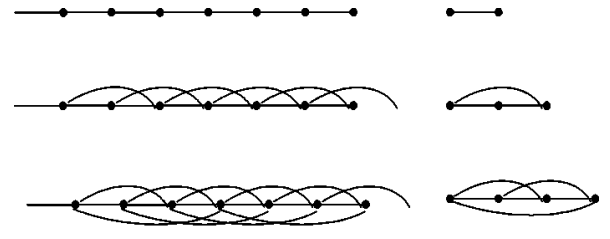

(a)

(b)

FIG. 1. A schematic representation of the construction of the infinite $g$-chain along the first (a) and second strategies (b).

The Tsallis proposal has motivated a large number of works aiming to certify its validity [12-14]. Many distinct models with long-range interactions have been investigated ever since, including spin models within mean-field, renormalization group, and Monte Carlo simulation approaches [15-17]. The present investigation considers one of the most simple long-range systems, for which numerical results of the thermodynamic properties - that take into account all energy levels of finite-size approximations-can be computed. The results discussed herein show that, despite the limitations imposed by the size of the considered systems, TS is verified for all analyzed thermodynamic functions with a satisfactory degree of accuracy.

The rest of this work is organized as follows. In Sec. II, we discuss the transfer matrix method (TM) used to evaluate the partition function and the free energy. In Sec. III we show that, for the free energy, TS is satisfied with high accuracy. The evaluation of the correlation function, the correlation length, and the critical temperature are developed in Sec. IV. In Sec. V, we discuss results for the entropy, specific heat, and magnetization, all of which agree with the proposed behavior within the TS framework. Section VI closes the work with our final remarks and conclusions.

\section{TRANSFER MATRIX FORMULATION OF THE PROBLEM}

As advanced in the Introduction, the long-range Ising chain is described by the Hamiltonian

$$
\mathcal{H}=-\sum_{i=0}^{\infty} \sum_{r=i+1}^{\infty} J_{r} \sigma_{i} \sigma_{i+r}-H \sum_{i=0}^{\infty} \sigma_{i}
$$

We consider two different ways to step over from a finite to an infinite chain with an infinite number of interaction constants, as illustrated in Fig. 1. In the first one, we start with an infinite 1 -chain, at generation $g=1$, with only firstneighbor interaction $J_{1}$, and add, successively, interactions $J_{2}, J_{3}, \ldots$. So, all interactions $J_{g}$ are introduced at the same $g$ th step of decoration, defining an infinite $g$-chain [see Fig. 1(a)]. In the second way, we start, at $g=1$, with a finite twospin chain and one $J_{1}$ interaction, and move onto generation $g=2$ by adding a third spin together with all new interactions that fit into the resulting array. This procedure can be repeated over and over: for a generic value $g$, we introduce one spin and $g$ new interactions mediated by $J_{r}, r=1,2, \ldots, g$, that link the $(g+1)$ th spin to all spins introduced in the previous generations [see Fig. 1(b)]. Of course the two proce- dures lead to the same system when $g \rightarrow \infty$ but, for finite values of $g$, the approximate results differ from each other.

In this work we introduce TM's that can describe interactions in both construction ways. This is done by $2 \times 2 \mathrm{TM}$ $M_{g, c}$, that describe finite chains of $N=g+c+1$ spins, encompassing all interactions among spins $g$ sites apart. This scheme differs from that in [8] where, at generation $g$, a $2^{g} \times 2^{g}$ TM $\hat{M}_{g}$ describes all the $g^{2}$ interactions among spins in two neighboring $g+1$ patches. The $g$-approximate partition function $Z_{g}$ for the infinite $g$-chain is obtained from the numerically evaluated largest eigenvalue $\Lambda_{g}$ of $\hat{M}_{g}$.

We consider first the situation $N=g+1$, illustrated in Fig. $1(\mathrm{~b})$, and define a $2 \times 2 \mathrm{TM} M_{g} \equiv M_{g, c=0}$, that describes all interactions within a patch limited by the spins $s_{0}$ and $s_{g}$. Within this definition, a partial trace, over all configurations involving spins $s_{i}, i \in[1, g-1]$ must be performed. This can be accomplished in a very efficient way if we make use of nonsquare TM's, as those used to describe spin models on hierarchical lattices $[18,19]$. This approach is simpler than the first one, since the TM's are smaller and the numerical evaluation of their largest eigenvalue is not required. So, starting with $g=1$ and restricting our discussion to the simpler situation when $H=0$, the quite obvious TM $M_{1}$ can be rewritten in the following way:

$$
M_{1}=\left(\begin{array}{ll}
a_{1} & b_{1} \\
b_{1} & a_{1}
\end{array}\right)=\left(\begin{array}{cccc}
a_{1} & b_{1} & 0 & 0 \\
0 & 0 & b_{1} & a_{1}
\end{array}\right)\left(\begin{array}{cc}
1 & 0 \\
0 & 1 \\
1 & 0 \\
0 & 1
\end{array}\right) \equiv P_{1} L_{1},
$$

where $a_{1}=b_{1}^{-1}=\exp \left(J_{1} / T\right)$. The expanded TM $P_{1}$ links spin $s_{0}$ to the pair $\left(s_{0}, s_{1}\right)$, while $L_{1}$ projects the pair $\left(s_{0}, s_{1}\right)$ to $s_{1}$, by performing a sum over values of $s_{0}$. Here, as well as throughout the work, we make use of the lexicographic order to associate a given spin configuration with the matrix element labels. This procedure is extended to all values of $g$, according to

$$
M_{g}=\left(\prod_{k=1}^{g} P_{k}\right) L_{g} \equiv R_{g} L_{g},
$$

where $L_{g}$ is a $2^{g+1} \times 2$ matrix whose elements $\left(L_{g}\right)_{i, j}=1$ for $i+j$ even, and $\left(L_{g}\right)_{i, j}=0$ for $i+j$ odd. The matrix elements of the $2^{k} \times 2^{k+1}$ TM's $P_{k}$ are recursively expressed by

$$
\left(P_{k}\right)_{i, j}= \begin{cases}\left(P_{k-1}\right)_{i, j} a_{k}^{(-1)^{j-1}} & \text { for } i \leqslant 2^{k-1} \\ \left(P_{k}\right)_{2^{k}-i+1,2^{k+1}-j+1} & \text { and } j \leqslant 2^{k} \\ & \text { for } 2^{k-1} \leqslant i \leqslant 2^{k} \\ 0 & \text { and } 2^{k} \leqslant j \leqslant 2^{k+1} \\ \text { otherwise, }\end{cases}
$$

where $a_{k}=\exp \left(J_{k} / T\right)$. In Eq. (7), each TM $P_{k}$ couples a group of spins $\left\{s_{0}, s_{1}, \ldots, s_{k-1}\right\}$ to $\left\{s_{0}, s_{1}, \ldots, s_{k-1}, s_{k}\right\}$, accounting for the interaction of spin $s_{k}$ with spins $s_{\ell}, \ell=0,1, \ldots, k-1$. Equation (8) shows that $P_{k}$ contains the same interactions of $P_{k-1}$ with the addition of the interaction $J_{k}$. Matrices $P_{k}$ are 
very sparse, but their products, condensed in the $2 \times 2^{g+1} \mathrm{TM}$ $R_{g}$, store in a very compact way, the information scattered among them.

The matrix elements of $R_{g}$ are Boltzmann weights to each spin configuration of a $g$-chain, ordered in a convenient way, so that the effect of successively increasing the length of the chain can be easily accounted for by matrix multiplication, i.e., $R_{g}=R_{g-1} P_{g}$. As $M_{g}$ is symmetric, $R_{g}$ has point inversion symmetry with respect to the central position. So, for a given value $g$, this property allows us to occupy only the first line of a $2 \times 2^{g+1}$ field to write down the values of matrix elements and use the second line as a buffer to store the elements of $P_{g}$ that give rise to $R_{g}$. Note that an infinite product of $M_{g}$ describes an infinite chain, where all spins within one $g$-patch interact with each other, but not with spins in other patches.

This matrix definition can be extended to describe the case illustrated in Fig. 1(a). Such chains can be regarded as being formed by $g$-patches of spins, with interaction among spins of neighboring patches. For example, taking $g=2$, the configuration in Fig. 1(a) is recovered from Fig. 1(b), by attaching to it other 2-chains and including the interaction $J_{2}$ between the midspins of neighboring patches. This corresponds to $c>0$, i.e., $N>g+1$.

Let us assume that we have evaluated $M_{g}$ and proceed to obtain $M_{g+1}$. If we omit the Boltzmann weight $a_{g+1}$ in Eq. (8), the resulting TM $\overline{P_{g+1}}$ takes into account the interactions between spin $s_{g+1}$ with spins $s_{\ell}, \ell=1,2, \ldots, g$, while $s_{0}$ and $s_{g+1}$ do not interact any longer. Hence, the TM $\overline{M_{g+1}}$ $\equiv R_{g} \bar{P}_{g+1} L_{g+1}=M_{g, c=1}$ describes two partially overlapping $g$-patches, with some interactions among spins of distinct patches. Further, for any situation where all weights $a_{k}, k$ $=g+1, g+2, \ldots, g+c$ have been neglected, $M_{g, c}=\bar{M}_{g+c}$ offers an exact description of chains constructed along the first way, valid also in the $c \rightarrow \infty$ limit.

The evaluation of $\overline{P_{k}}, k=g+1, \ldots, g+c$, required for building $\overline{R_{k}}$ and $M_{g, c}$, can be circumvented. This is easily seen for the matrix $M_{g, 1}$. If $a_{g+1}$ is not included in Eq. (8), the elements of $\overline{P_{g+1}}$ are the same as those of $P_{g}$, but each one of them appears twice as much as in $P_{g}$. So, in the evaluation of $\overline{R_{g+1}}=R_{g} \overline{P_{g+1}}$, at least two elements of $R_{g}$ will be multiplied by the same factor. Due to the lexicographic labeling used herein, we identify that this fact happens for all pairs $\left(R_{g}\right)_{i, j}$ and $\left(R_{g}\right)_{i, 2^{g}+j}, j=1,2, \ldots, 2^{g}$. They correspond exactly to the same spin configuration except by the flipping of the spin $s_{0}$. As the interaction energy of the spins $s_{1}, s_{2}, \ldots, s_{g}$ with $s_{g+1}$ in $\overline{P_{g+1}}$, for these two configurations, is the same, the information in $\overline{R_{g+1}}$ can be condensed in

$$
R_{g, 1}=R_{g} Q_{g} P_{g}
$$

$Q_{g}$ is a $2^{g+1} \times 2^{g}$ matrix defined by

$$
\left(Q_{g}\right)_{i, j}= \begin{cases}1 & \text { for } i=j \text { or } i=j+2^{g} \\ 0 & \text { otherwise }\end{cases}
$$

that performs the sum of $\left(R_{g}\right)_{i, j}$ and $\left(R_{g}\right)_{i, 2^{g}+j}$ prior to the multiplication by $P_{g}$. This definition can be extended in a straightforward way, so that all interactions in a chain with
$N=g+c+1$ spins and interactions among $g$ nearest neighbors can be cast into

$$
M_{g, c}=\left(\prod_{k=1}^{g} P_{k}\right)\left(Q_{g} P_{g}\right)^{c} L_{g} \equiv R_{g, c} L_{g}
$$

It is important to observe that the matrix elements of $R_{g, c \neq 0}$ are no longer single Boltzmann weights corresponding to a particular spin configuration.

The free energy per spin, $f_{g, c}=-T \ln \left(Z_{g, c}\right) / N$, and the TS free energy $\tilde{f}_{g, c}=f_{g, c} / \tilde{N}$ follow from the partition function

$$
Z_{g, c}=2 \lambda_{g, c}^{+}=2\left[\left(M_{g, c}\right)_{1,1}+\left(M_{g, c}\right)_{1,2}\right]=\sum_{i, j}\left(R_{g, c}\right)_{i, j}
$$

This scheme can be easily enlarged to include the more general situation when $H \neq 0$, required for the evaluation of the magnetization $m$. The basic relations are still valid, with the exception of the matrix element point inversion symmetry with respect to the central position. The only consequence is that it demands twice as many variables to store the fielddependent energy levels.

\section{TSALLIS SCALING FOR THE FREE ENERGY}

Let us now discuss results that show a remarkable precision in the collapse of the Tsallis rescaled free energy for the Ising chain. The partition function $Z_{g, c}$ was evaluated for different values of $\alpha$, taking $g=1,2, \ldots, 24$ and $c=1,2, \ldots$, 200 , performing this way several tests for the validity of TS. For the sake of clearness, we show some of our results are shown in Figs. 2 and 3 for selected values of $g$ and $c$ only.

Figure 2(a) shows results for $f_{g, c}(T)$, when $\alpha=0.5$ and 2.0, for $c=0$ and several values of $g$. Two features can easily distinguish nonextensive $(\alpha<1)$ from extensive $(\alpha>1)$ systems: (i) the values of $f$ converge with $g$ for the latter but not for the former; (ii) the width of the low-temperature plateau increases with $g$ for the nonextensive case while, in the extensive situation, despite a discrete increase, it rapidly converges to a fixed length. For $\alpha=0.5$, when $g \rightarrow \infty$ this plateau extends itself to $T \rightarrow \infty$, corresponding to a single ordered phase of zero entropy [6]. Figure 2(b) shows the influence of increasing $c$ on $f$ when $\alpha=1.2$, for a fixed value of $g$, indicating that a slow convergence is achieved. The same kind of dependence on $c$, for fixed $g$, is observed for nonextensive cases.

Performing TS on $f$ leads to the curves shown in Fig. 3 for $\tilde{f}_{g, c}(\widetilde{T}) \times \widetilde{T}$. An important finding is that the correct scaling, when $c \neq 0$, requires that the total number of components $N=g+c+1$ in Eq. (2) be replaced by the range of the interaction $g$. In Fig. 3(a) we show, for $\alpha=0.5$, the influence of both $g$ and $c$. Rather small values for $g$ have been chosen to better display the effect of increasing it. We note that curves for distinct values of $g$ are gathered together for fixed values of $c$ rather than the other way around. This shows clearly that (i) TS is directly related to the range $g$ of interactions, not to the number of constituents $N$; (ii) for small 

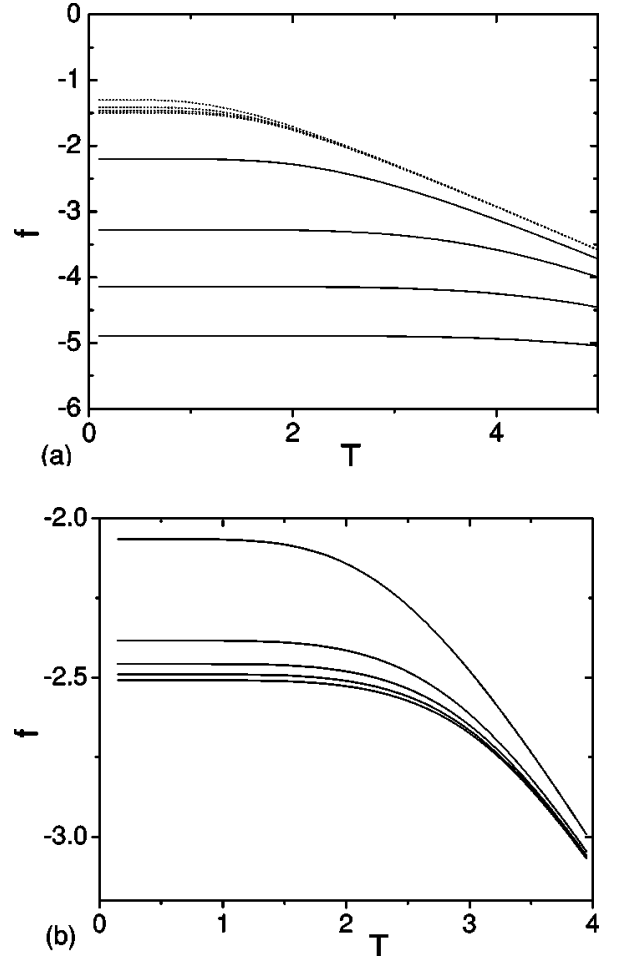

FIG. 2. (a) Free energy $f$ for $\alpha=0.5$ (solid) and 2.0 (dashed), when $c=0$ and $g=5,10,15$, and 20; $f$ decreases when $g$ increases. Convergence is observed only for the extensive case. (b) The same as in (a), for $\alpha=1.2$ and fixed $g=12$. $f$ decreases when $c$ increases from 0 to $20,40,60$, and 80 .

values of $g$, different values of $c$ are treated by TS as different systems. As expected, for larger values of $c$, the curves are less dispersed.

Figure 3(a) also shows, in the inset, results for $\alpha=1.2$ and for much larger values of $g$ and $c$. Eight curves are superimposed, attesting a very good convergence, which is very clearly shown in Fig. 3(b), where we draw the difference between two curves of the inset of Fig. 3(a) as a function of $g$ and $c$. Holding $g$ constant, the difference $\widetilde{f}_{g, c+\delta}(\widetilde{T})$ $-\tilde{f}_{g, c}(\widetilde{T})$ monotonically decreases with respect to $\widetilde{T}$. The curves differ by less than 0.0035 for the whole temperature range. As $\delta=10$, this indicates that the discrepancy by increasing $c$ by 1 is less than $0.0004 \%$. The behavior found for other values of $\alpha$ is much the same as those shown here, e.g., for $\alpha=0.5$, the discrepancies are less than $0.0008 \%$ for the same values of $g$ and $c$ used in Fig. 3 .

On the other hand, holding $c$ fixed, $\tilde{f}_{g+\delta, c}(\widetilde{T})-\tilde{f}_{g, c}(\widetilde{T})$ is bounded by the same value above. Since now $\delta=2$, the discrepancy by increasing $g$ by 1 is less than $0.002 \%$. We also observe that the curves go through a maximum at nonzero value of $\widetilde{T}$. The position of this maximum on the $\widetilde{T}$ axis, indicated by an arrow in the inset of Fig. 3(a), is close to the peak of the specific heat, as will be discussed later. Although very small, the inclusion of new interaction constants causes a nontrivial effect in the free energy, that might be useful when exploring the critical properties of the model. For $\alpha$ $=0.5$, the distance between the curves gets roughly doubled, much like when $g$ is fixed. Their shape is now much closer to
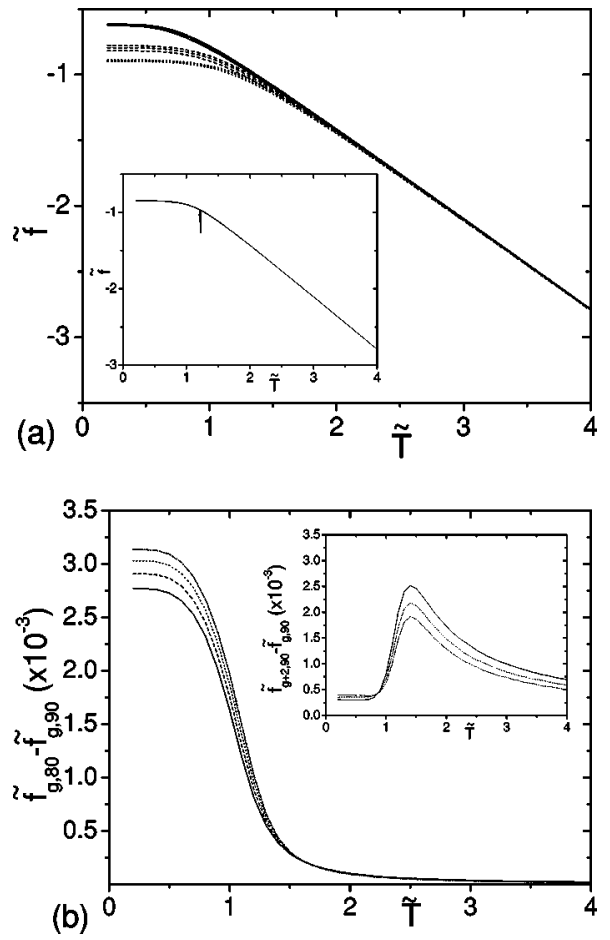

FIG. 3. (a) TS free energy $\tilde{f}$ for $\alpha=0.5$ when $c=0$ (solid), 10 (dashed), and 50 (dotted). Three curves in each group were obtained for $g=6,8$, and 10. Inset: superposition of eight curves for $\alpha=1.2$, when $g=18,20,22,24$ and $c=80,90$. (b) For $\alpha=1.2$, differences $\widetilde{f}_{g, 80}(\widetilde{T})-\widetilde{f}_{g, 90}(\widetilde{T})$, when $g=18,20,22,24$. Magnitude increases with $g$. Inset: differences $\tilde{f}_{g, 90}(\widetilde{T})-\tilde{f}_{g+2,90}(\widetilde{T})$ when $g=18,20,22$. Here the magnitude decreases with $g$.

those of $\tilde{f}_{g, c+\delta}(\tilde{T})-\tilde{f}_{g, c}(\widetilde{T})$, however superimposed to a small peak that rapidly vanishes upon increasing $g$.

Still better convergence can be achieved by increasing the values of $g$ and $c$ but, in our opinion, the precision we attained is sufficient to attest the validity of TS, for both nonextensive and extensive values of $\alpha$.

\section{CORRELATION FUNCTION AND THE CRITICAL TEMPERATURE}

The correlation function between spins placed $r$ sites apart depends on both the range of interactions and the size of the system. Restricting to the situation where $c=0$, we define a $g$-dependent correlation function between the first and the $r$ th spins along the chain $C_{g}(r ; T)=\left\langle\sigma_{1} \sigma_{r}\right\rangle_{g}, r=1, \ldots, g$. Using the fact that the row and column labels of the matrix elements of $P_{k}$ are defined according to the lexicographic order, it is straightforward to show that $C_{g}(r ; T)$ can be easily expressed in terms of the matrix elements of $R_{g}$ as

$$
C_{g}(r ; T)=\frac{1}{Z_{g}} \sum_{i, j}\left[\underline{R}_{g} L_{g, r}\right]_{i, j} \equiv \frac{1}{Z_{g}} \sum_{i, j}\left[\underline{P}_{1}\left(\prod_{k=2}^{g} P_{k}\right) L_{g, r}\right]_{i, j},
$$

where 


$$
\begin{gathered}
\underline{P}_{1}=\left(\begin{array}{llll}
a_{1} & b_{1} & 0 & 0 \\
0 & 0 & -b_{1} & -a_{1}
\end{array}\right), \\
\left(L_{g, r}\right)_{i, j}=-1^{q_{g, r}(j)}, \quad q_{g, r}(j)=L\left[\frac{j-1}{2^{g-r}}\right],
\end{gathered}
$$

with $L[x] \equiv$ largest integer in $x$.

The definition of $C_{g}(r ; T)$ can be extended to more general situations $C_{g, c}(i, i+r ; T)$, where $c>0, r>g$, and the $i$ th spin must not necessarily sit on the first place of the chain. However, as the expression for $C_{g, c}(i, i+r ; T)$ in terms of the matrix elements of $P_{k}$ 's is much more complex and the essential behavior of the spin correlation function can be obtained from Eq. (13), let us proceed with the discussion of the results obtained by this simpler situation. It can be better accomplished if we refer to a series of graphics for $C_{g}(r ; T) \times r$ and $C_{g}(r ; T) \times g$ shown in Fig. 4. In Fig. 4(a), we take the limit situation $\alpha=0$, for which the spins should be aligned at all temperatures when $N \rightarrow \infty$. For $T=10$, when the values of $C_{g}(r ; T) \times r$ are relatively scattered, we see that they are almost insensitive with respect to $r$ for a fixed $g$ and, for fixed $r$, they increase monotonically with $g$. The qualitative features of this picture do not change with the temperature, and are consistent with the expected behavior, namely $C_{g}(r ; T)=1, \forall r$ when $N \rightarrow \infty$.

Going far inside the extensive region, we show in Fig. 4(b) the behavior for $\alpha=10$. The several curves for $C_{g}(r ; T) \times g$, at $T=0.5$, show a reverse situation to the one obtained when $\alpha=0$ : they are almost insensitive with respect to $g$ for a fixed $r$ while, for fixed $g$, they decay exponentially with $r$. This $T$-independent behavior is in accordance with the expected absence of ordering at any finite temperature.

When $1<\alpha \leqslant 2$, a phase transition at finite temperature occurs. The behavior of $C_{g}(r ; T) \times r$ when $\alpha=1.2$ for two distinct temperatures is drawn in Figs. 4(c) and 4(d). In both cases we see that, for a fixed value of $g, C_{g}(r ; T)$ clearly decays with $r$, especially when $r \sim g$. For a low value of $T$ [Fig. 4(c)], we note that $C_{g}(r=g ; T)$ initially decays with $r$, but then this trend is reversed. On the other hand, for a larger value of $T$ [Fig. 4(d)], $C_{g}(g ; T)$ keeps its decreasing behavior until the largest value of $g$. Of course we cannot anticipate whether, for this particular value, the curve will reverse its derivative. Nevertheless, these results strongly suggest that a $T$-dependent twofold behavior should emerge for that interval of $\alpha$, reflecting the emergence or absence of long-range spin ordering.

The observed behavior for $C_{g}(r ; T)$ suggests a scheme leading to finite-size estimates of the critical temperature of the system. For a given value of $g$, we can indicate that the system will develop long-range order at a particular temperature $T$ if $C_{g}(g ; T)>C_{g-1}(g-1 ; T)$. This inequality is only a sufficient condition for the existence of long-range ordering when $g \rightarrow \infty$, but it can be used to define a series of $g$-dependent critical temperatures $T_{c, g}$, which constitute lower bounds for the actual critical temperature of the chain. Thus it is natural to define $T_{c, g}$ and $T_{c}$ by

$$
C_{g}\left(g ; T_{c, g+1}\right)=C_{g+1}\left(g+1 ; T_{c, g+1}\right) \text {, }
$$
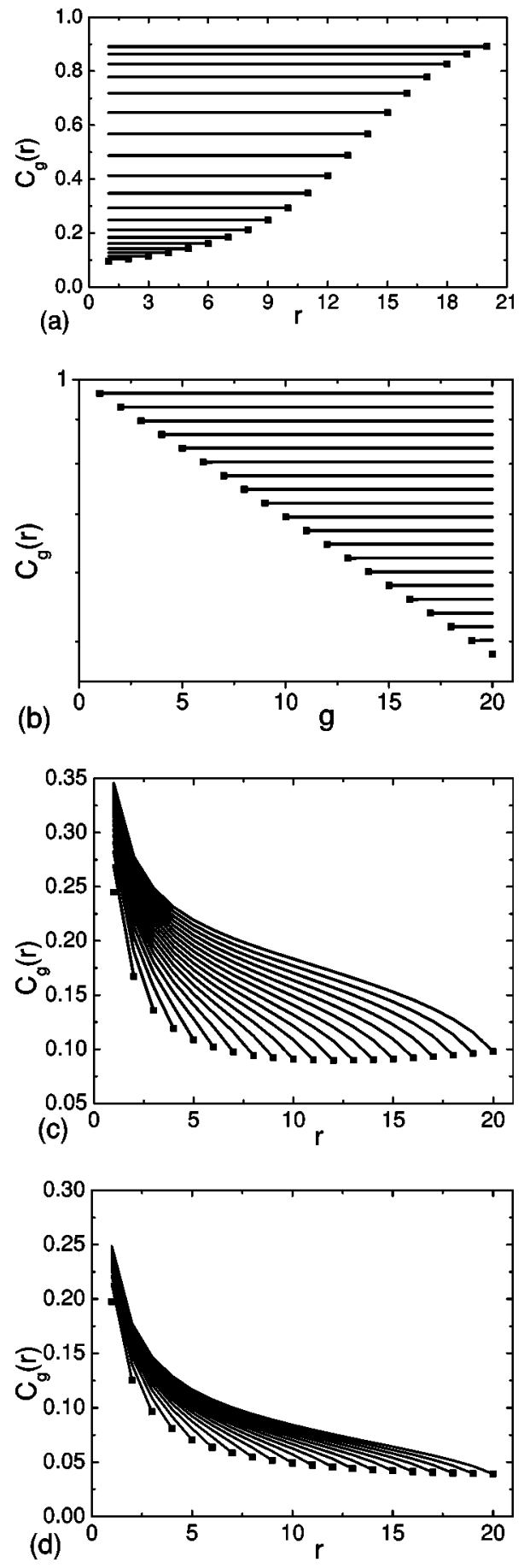

FIG. 4. (a) Correlation function $C_{g}(r, T) \times$ distance $r$ when $\alpha$ $=0$ and $T=10$. Each line corresponds to a value of $g$ from 1 (bottom) to 20 (top) and the squares indicates $C_{g}(g, T)$. (b) Correlation function $C_{g}(r, T)$ in logarithm scale $\times g$ when $\alpha=10$ and $T=0.5$. Each line corresponds to a value of $r$ from 1 (top) to 20 (bottom). As in case (a), the squares indicate $C_{g}(g, T)$. (c) The same as (a) for $\alpha=1.2$ and $T=4.5$. (d) The same as (c) for $T=5.0$.

$$
T_{c}=\lim _{g \rightarrow \infty} T_{c, g} .
$$

If we use Eq. (13) for $r=g$, it is possible to express $C_{g}(g ; T)$ in terms of the eigenvalues of $M_{g}$ as $C_{g}(g ; T)$ $=\lambda_{g}^{-} / \lambda_{g}^{+}$. Note further that the correlation length for a chain 
TABLE I. Values of the critical temperature $T_{c, g}$ and the Tsallis scaled counterpart $\widetilde{T}_{c, g}$ for $\alpha=1.2$ and $\alpha=1.8$, for odd $g$ from 1 to 23 . The extrapolated values obtained by the VBS procedure using odd and even values of $g$ are included in the last row. For comparison, the reported values of $T_{c}$ in Ref. [8] are, respectively, 10.84 and 2.43 .

\begin{tabular}{ccccc}
\hline \hline$g$ & \multicolumn{2}{c}{$\alpha=1.2$} & \multicolumn{2}{c}{$\alpha=1.8$} \\
& $T_{c, g}$ & $\tilde{T}_{c, g}$ & $T_{c, g}$ & $\tilde{T}_{c, g}$ \\
\hline 1 & 1.3556580 & 0.8229839 & 0.8388462 & 0.5475270 \\
3 & 2.3629378 & 1.0688599 & 1.2011865 & 0.6536522 \\
5 & 2.9514932 & 1.1778344 & 1.3667507 & 0.7002222 \\
7 & 3.3616308 & 1.2444814 & 1.4699165 & 0.7301505 \\
9 & 3.6731505 & 1.2909930 & 1.5432409 & 0.7521076 \\
11 & 3.9224949 & 1.3259833 & 1.5993359 & 0.7693643 \\
13 & 4.1292676 & 1.3536242 & 1.6443204 & 0.7835166 \\
15 & 4.3051803 & 1.3762246 & 1.6815968 & 0.7954662 \\
17 & 4.4577656 & 1.3951828 & 1.7132407 & 0.8057728 \\
19 & 4.5921416 & 1.4114038 & 1.7406053 & 0.8148082 \\
21 & 4.7119372 & 1.4255034 & 1.7646193 & 0.8228320 \\
23 & 4.8198144 & 1.4379177 & 1.7859455 & 0.8300324 \\
VBS extrapolation & 10.713270 & 1.785545 & 2.474799 & 1.099911 \\
\hline \hline
\end{tabular}

composed of patches described by the matrix $M_{g}$, as mentioned in Sec. II, is given by

$$
\xi_{g}=\frac{g}{\ln \left(\lambda_{g}^{+} / \lambda_{g}^{-}\right)}=-\frac{g}{\ln \left[C_{g}\left(g ; T_{c, g}\right)\right]} .
$$

So, combining Eqs. (15) and (16), it is possible to rewrite the criterion for the finite-size estimates for the critical temperature $T_{c, g}$ as

$$
\frac{\xi_{g}\left(T_{c, g+1}\right)}{g}=\frac{\xi_{g+1}\left(T_{c, g+1}\right)}{g+1} .
$$

Finally, we recall that Eq. (17) coincides with the expression used by Glumac and Uzelac [8], derived within the method of finite-range scaling, to evaluate finite-size estimates for the critical temperatures. The steps leading to Eq. (17) use no scaling arguments, and it is rather amazing to obtain the same expression working within two different frameworks.

Series of very precise values of $T_{c, g}$ for $\alpha=1.2$ and 1.8 are shown in Table I. For $\alpha=1.2$, we observe that the values in Table I agree with the illustrations in Figs. 4(c) and 4(d) as $4.5<T_{c, g=20}<5.0$. Using Padé approximants or other similar techniques, it is possible to extrapolate the finite series results for a limit value $T_{c}$, which has been inserted in the last row in the bottom of Table I. A comparison of the results in Table I shows that our results for $T_{c, g}$ are smaller than the corresponding values reported in Ref. [8]. This difference is due to the smaller amount of interaction energy that is included in one matrix $M_{g}$. Nevertheless, with the help of the Vanden Broeck and Schwartz (VBS) extrapolation procedure used in [8], we are led to values for $T_{c}$ that reproduce those reported in the literature with an accuracy of 1.2 and $1.6 \%$ for $\alpha=1.2$ and 1.8. In Table I, we also include values for $\widetilde{T}_{c, g}=T_{c, g} / \tilde{N}$ and $\widetilde{T}_{c}=T_{c}(\alpha-1) / \alpha$.

\section{DERIVATIVES OF THE FREE ENERGY}

As introduced in Eq. (4), TS foresees for the intensive quantities $\tilde{s}, \tilde{c}$, and $\tilde{m}$ a different behavior from the one for the free energy (1), that has been explored in Sec. III. This is related to the fact that they measure properties of the internal degrees of freedom of the system that cannot increase beyond a saturation point even with the addition of energy from the long-range interactions. In any case, as their thermodynamic conjugate intensive variables scale with $\widetilde{T}$, the summands in any Legendre transform have an overall correct scaling.

We have investigated the behavior of derivatives of the free energy along two different paths: by numerically deriving expressions (8) and (11), or by working out analytical expressions from the Boltzmann weights stored in the matrix elements. The results agree with each other within the expected numerical accuracy. The second strategy requires further analytical work, but its results are more stable and avoid rounding off errors resulting from differences between two quite close values. Because of this, our discussion is based on results along the second path. For any of the three quantities, it is straightforward to set up expressions for the new matrix elements that describe the effects of taking the derivative of the Boltzmann weights with respect to the temperature and the magnetic field. For instance, in the case of the magnetization, we obtain

$$
m(T, H)=\frac{1}{Z_{g}(T, H)} \sum_{i, j} p_{g}(j)\left(R_{g}\right)_{i, j} d^{p_{g}(j)},
$$

where 


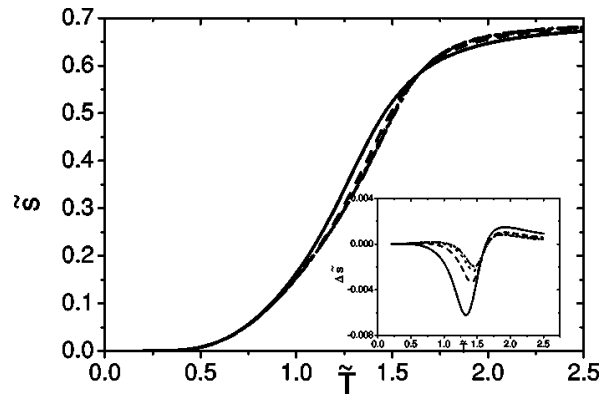

FIG. 5. TS entropy $\widetilde{s}$ for $\alpha=0.5, H=0, c=200$, and different values of $g$ : 10 (solid), 16 (dashed), 20 (dotted), 22 (dot-dashed), and 23 (dash-dot-dotted). Inset: the difference $\Delta \widetilde{s}$ for the same parameters of the main set.

$$
\begin{gathered}
Z_{g}(T, H)=\sum_{i, j}\left(R_{g}\right)_{i, j} d^{p_{g}(j)}, \quad p_{g}(j)=\sum_{r=1}^{g} q_{g, r}(j), \\
d=\exp (H / T) .
\end{gathered}
$$

Results for the TS entropy $\tilde{s}$ are shown in Fig. 5. Convergence has been checked for both situations explored in Sec. III: holding $c$ fixed and letting $g$ change or the other way around. Let us focus the discussion on the first possibility, as it is more relevant for the purpose of checking the TS validity. We show in the main set, for $H=0, \alpha=0.5$, and $c=200$, five curves for $g=10,16,20,22$, and 23. The two curves for lower values of $g$ are still a bit apart from those for $g=22$ and 23 , which collapse with each other with a precision of the order of $10^{-3}$, as blown up in the inset for the difference between the values of $\tilde{s}$ for the successive values of $g, \Delta \tilde{s}$
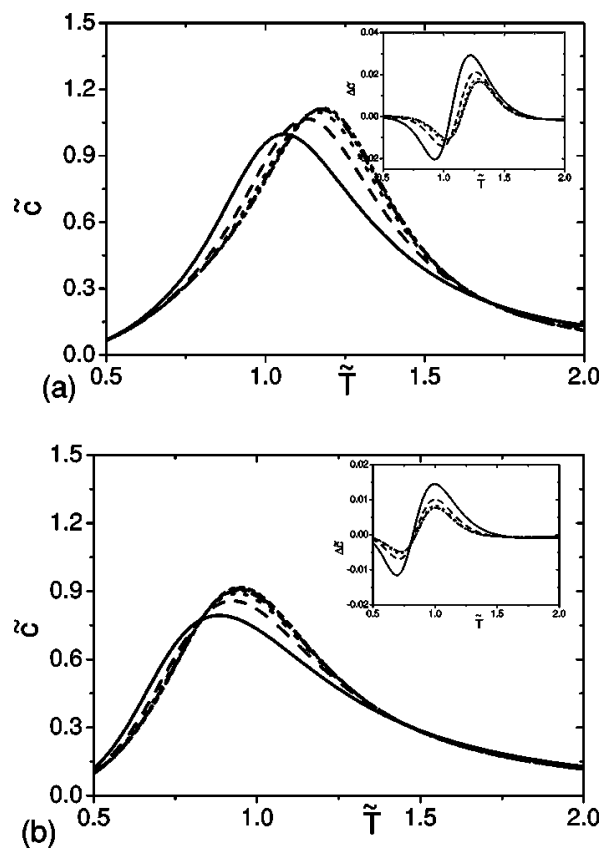

FIG. 6. (a) TS specific heat $\tilde{c}$ for $\alpha=1.2, H=0, c=90$, and different values of $g$ : 10 (solid), 16 (dashed), 20 (dotted), 22 (dotdashed), and 23 (dash-dot-dotted). Inset: the difference $\Delta \tilde{c}$ for the same parameters of the main set. (b) The same as (a), for $\alpha=1.8$.

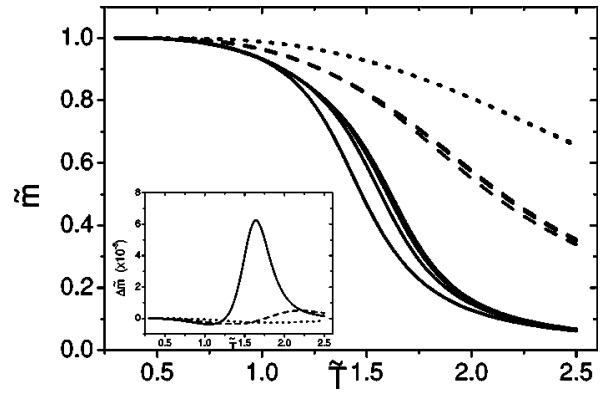

FIG. 7. TS magnetization $\tilde{m}$ for $\alpha=0.5, c=150$, and different values of field $H$ : 0.05 (solid), 0.3 (dashed), and 0.8 (dotted). For each value of $H$, there are five curves for $g=10,16,20,22,23$. Inset: the difference $\Delta \tilde{m}$ for the same parameters of the main set with the respective symbols when $g=22$ and $\delta=1$.

$=\left(\tilde{s}_{g+\delta}-\tilde{s}_{g}\right) / \delta$. The attained precision of convergence for this value of $g$ is somewhat smaller than that for the free energy, but this is not unexpected, as the derivative operator usually enhances differences between similar objects. The same kind of behavior is observed for other values of $c$. Only for smaller values $c<50$ is it possible to detect a noticeable increase in the discrepancies between the curves for different values of $g$. As we can expect from Fig. 2, if we draw the corresponding curves of $s(T, H, N) \times T$, we observe that they do not collapse at low values of $T$, but move to the right with increasing value of $N$.

Essentially the same kind of situation is observed for the curves of the TS specific heat $\tilde{c}$ shown in Figs. 6(a) and 6(b), for $\alpha=1.2$ and 1.8, $c=90$, and the same values for $g$. They go through a rather smooth maxima and then decrease with $\widetilde{T}$. We observe that the maxima of the curves occur at $\widetilde{T}_{M}$, which are smaller than $\widetilde{T}_{c}$ in Table I. For $\alpha=1.2$ and 1.8 we have, respectively, $\widetilde{T}_{M} / \widetilde{T}_{c} \simeq 0.66$ and 0.87 . Similar discrepancies between the specific-heat maxima and the critical temperature have been reported by Nagle and Bonner [5], who refer to a very slow convergence of the specific-heat maxima towards the values of the critical temperature obtained by the behavior of other thermodynamic properties. In the insets of Fig. 6 we show, for each set of $\alpha$, differences $\Delta \widetilde{c}=\left(\widetilde{c}_{g+\delta}\right.$ $\left.-\widetilde{c}_{g}\right) / \delta$. The progressive displacement of the maxima to the

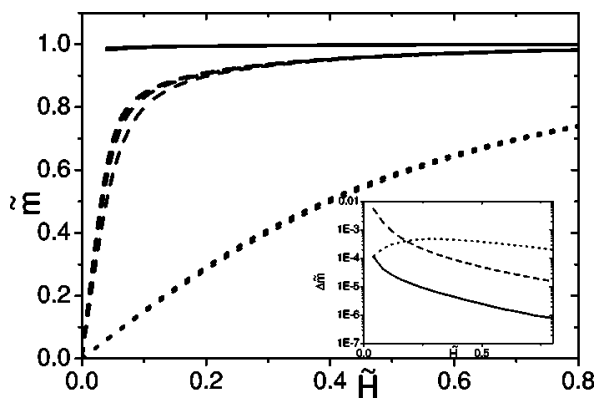

FIG. 8. TS magnetization $\tilde{m}$ for $\alpha=1.8, c=150$, and different values of field $T$ : 0.6 (solid), 1.0 (dashed), and 2.0 (dotted). For each value of $T$, there are five curves for $g=10,16,20,22,23$. Inset: the difference $\Delta \tilde{m}$ for the same parameters of the main set with the respective symbols when $g=22$ and $\delta=1$. 
right is clearly enhanced, which indicates the trend of the curve maxima motion reported above. As expected, a slight decrease of the relative precision among the curves, as compared to that for $\tilde{f}$ and $\tilde{s}$, is observed for the entire range of reduced temperature, being more relevant for the smaller value of $\alpha$.

Finally, in Fig. 7 and 8 we illustrate the behavior of $\tilde{m}$ with respect to both $\widetilde{T}$ and $\tilde{H}$. These curves are important to show the validity of the TS conjecture with respect to the magnetic field. In Fig. 7, curves are drawn for $\alpha=0.5$ and three different values of $\tilde{H}$, while in Fig. 8 we consider $\alpha$ $=1.8$ and three values of $\tilde{T}$. For both cases we use the same values of $g$ as before, and $c=150$. The quality of the collapsing of the curves depends slightly on the values of $H$ and, as in Fig. 6, on the value of $\alpha$. However, the precision measured by $\Delta \tilde{m}=\left(\tilde{m}_{g+\delta}-\tilde{m}_{g}\right) / \delta$ is still very high $\left(<10^{-2}\right)$ for both values of $\alpha$ when $g=22$ and 23 .

\section{CONCLUSIONS}

In this work, we presented a thorough investigation of the thermodynamic properties of the Ising chain, focusing on the set scaling properties proposed by Tsallis.

The investigation was carried out with the help of an efficient procedure to obtain $Z(T, N=g+c+1)$ of long-range Ising chains. It requires only a storage space and small TM, avoiding the necessity of eigenvalue evaluation. The CPU time required for its implementation grows exponentially with $g$ and linear with $c$, while the storage necessity increases exponentially with $g$ and is insensitive to the value of $c$. The results were obtained with a double-precision FORTRAN code implemented on a common desk computer.

The comparison with similar results reported by other authors and the high degree of accuracy indicate that the proposed procedure is reliable. This is evidenced, in particular, by the reproduction of a criterion for the critical temperature based on the analysis of the correlation function, which was previously obtained within the finite-range scale framework, together with the actual values for $T_{c}$ that we have obtained.

We have shown that all thermodynamic properties of the chain, such as the free energy, entropy, specific heat, and magnetization, satisfy TS with a high degree of accuracy for both nonextensive $(\alpha<1)$ and extensive $(\alpha>1)$ regimes. This agrees with other analyses of long-range systems, which have been carried out with the help of such other approaches as the renormalization group and Monte Carlo methods. Still better precision in the results can be achieved by increasing the values of $g$ and $c$, but our purpose of showing the validity of TS is fully completed by the present contribution.

\section{ACKNOWLEDGMENTS}

We acknowledge E. P. Borges for useful discussions and remarks.
[1] F. J. Dyson, Commun. Math. Phys. 12, 91 (1969).

[2] J. Frölich and T. Spencer, Commun. Math. Phys. 84, 87 (1982).

[3] J. L. Cardy, J. Phys. A 14, 1407 (1981).

[4] M. Aizenman, J. T. Chayes, L. Chayes, and C. M. Newman, J. Stat. Phys. 50, 1 (1988); M. Aizenman and R. Fernández, Lett. Math. Phys. 16, 39 (1988).

[5] J. F. Nagle and J. C. Bonner, J. Phys. C 3, 352 (1970).

[6] M. E. Fisher, S. K. Ma, and B. G. Nickel, Phys. Rev. Lett. 29, 917 (1972).

[7] J. L. Monroe, R. Lucente, and J. P. Hourlland, J. Phys. A 23, 2555 (1990).

[8] Z. Glumac and K. Uzelac, J. Phys. A 22, 4439 (1989), 26, 5267 (1993).

[9] S. A. Cannas and A. C. N. de Magalhães, J. Phys. A 30, 3345 (1997).
[10] C. Tsallis, Braz. J. Phys. 29, 1 (1999).

[11] P. Jund, S. G. Kim, and C. Tsallis, Phys. Rev. B 52, 50 (1995); C. Tsallis, Fractals 3, 541 (1995).

[12] F. Tamarit and C. Anteneodo, Phys. Rev. Lett. 84, 208 (2000).

[13] V. Latora, A. Rapisarda, and C. Tsallis, Phys. Rev. E 64, 056134 (2001)

[14] H. H. A. Rego, L. S. Lucena, L. R. da Silva, and C. Tsallis, Physica A 266, 42 (1999).

[15] S. A. Cannas, Phys. Rev. B 52, 3034 (1995).

[16] S. A. Cannas and F. A. Tamarit, Phys. Rev. B 54, R12 661 (1996).

[17] S. A. Cannas, A. C. N. de Magalhães, and F. A. Tamarit, Phys. Rev. B 61, 11521 (2000).

[18] R. F. S. Andrade, Phys. Rev. E 61, 7196 (2000).

[19] R. F. S. Andrade and S. T. R. Pinho, Eur. Phys. J. B 34, 343 (2000). 